\title{
Influence of anisotropic next-nearest-neighbor hopping on diagonal charge-striped phases
}

\author{
V. Derzhko \\ Institute of Theoretical Physics, University of Wrocław, \\ pl. Maksa Borna 9, 50-204 Wrocław, Poland \\ e-mail: derzhko@ift.uni.wroc.pl \\ May 3, 2019
}

\begin{abstract}
We consider the model of strongly-correlated system of electrons described by an extended Falicov-Kimball Hamiltonian where the stability of some axial and diagonal striped phases was proved. Introducing a next-nearest-neighbor hopping, small enough not to destroy the striped structure, we examine rigorously how the presence of the next-nearest-neighbor hopping anisotropy reduces the $\pi / 2$-rotation degeneracy of the diagonal-striped phase. The effect appears to be similar to that in the case of anisotropy of the nearest-neighbor hopping: the stripes are oriented in the direction of the weaker next-nearest-neighbor hopping.
\end{abstract}

\section{Introduction}

Experimentally, the presence of the striped structures in materials exhibiting high-temperature superconductivity is well-known [1, 2]. Theoretical investigations of the stability and properties of striped phases are performed mainly with approximate methods. Usually, the Hubbard-like or $t-J$-like models, 'as the standard models applied to explain the effects in strongly correlated systems, are considered 'for this purpose (see for example a review 3]). Unfortunately, due to the tiny energy differences between the energies of compared phases, the methods that are applied to study the stability are not reliable. That is why some simpler models (see Refs. 4, 5, 6, 7]) are considered in the investigations of striped-structures stability and properties.

In the recent paper [8] we addressed the issue of striped-order formation in the systems of strongly'correlated quantum particles described by extended Falicov-Kimball models. We showed that at halffilling and in the strong-coupling regime some axial and diagonal striped phases are stable. In comparison to the standard spinless Falicov-Kimball model (like in Ref. 9, 10]) the model in Ref. [8] was augmented by a direct Ising-like nearest-neighbor (n.n.) attractive interaction between the immobile particles, in order to allow for segregated phases. Changing the intensity of the Ising-like n.n. interaction, the system is driven from a crystalline (chessboard) phase to a segregated phase, via quasi-onedimensional striped phases. This conclusion was reached for two kinds of hopping particles: fermions and hard-core bosons.

The obtained results enable us to continue the study of the properties of striped phases. One of the interesting questions, the influence of n.n. anisotropy of hopping intensity on axial striped phases, was investigated by means of the Hartree-Fock method in [3, 11. In Ref. [12, at the regime where stripes are stable, we have proved rigorously that for both systems, of hopping fermions and hard-core bosons, an arbitrarily small anisotropy of n.n. hopping orients the axial striped phases in the direction of a 


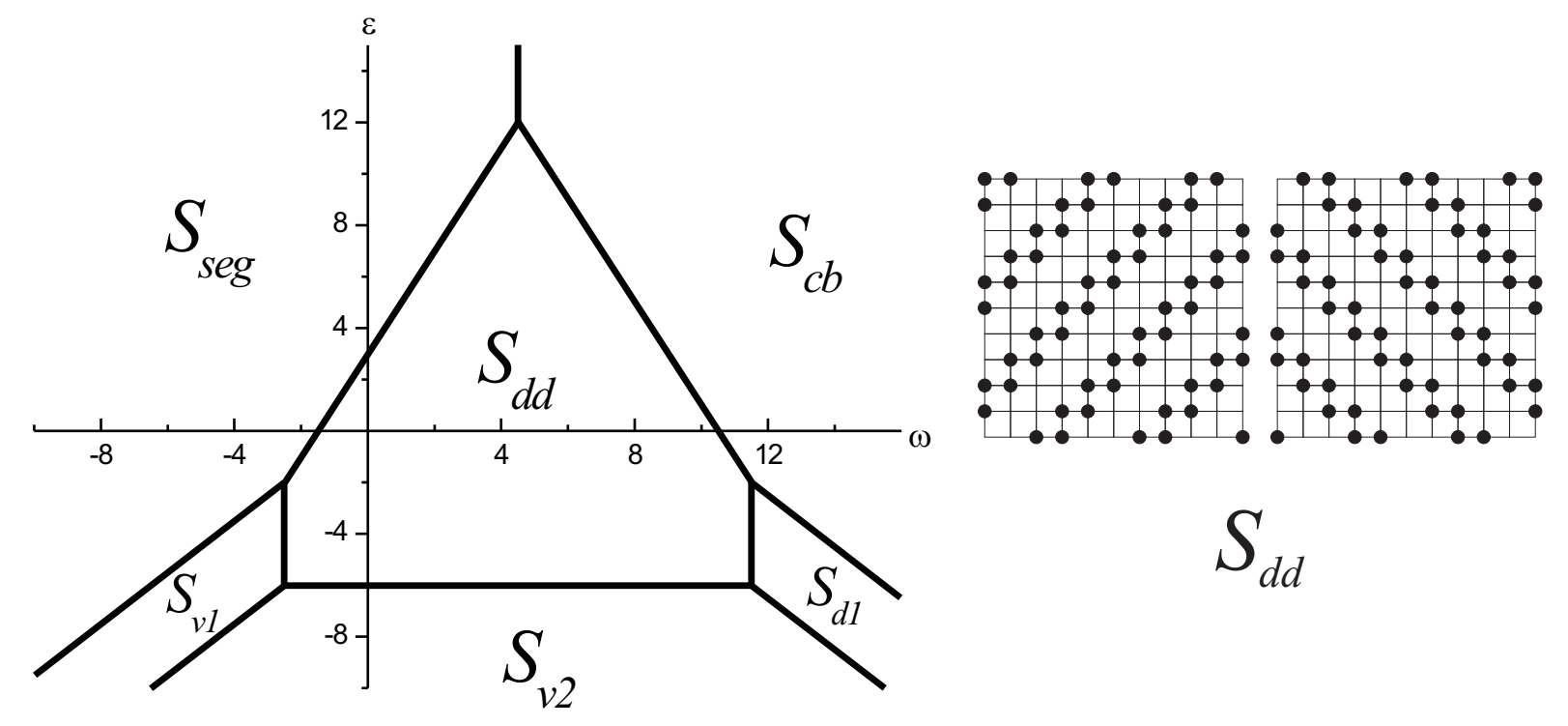

Figure 1: The phase diagram obtained in 8, containing a diagonal-striped phase $\mathcal{S}_{d d}$, at $\mu=0$, in the plane $(\omega, \varepsilon)$. In this paper $\omega$ is given by $W=-2 t^{2}+\omega t^{4}$, see (3), and $\varepsilon=0$. The representative configurations (up to translations) of phase $\mathcal{S}_{d d}$ are shown on the right. For more details see [8].

weaker hopping. We noted also the tendency of the phase diagrams for different statistics to become similar, even for a weak anisotropy of n.n. hopping.

The analogous, arising naturally, question is how the anisotropy of the next-nearest-neighbor (n.n.n.) hopping influences the degeneracy of diagonal-striped phases. To answer this question, in this paper, we use the same techniques as in [12. Specifically, we investigate the influence of n.n.n. hopping on the phase $\mathcal{S}_{d d}$, whose stability was proved for fermions in [8] (in Fig. 1] we reproduce the phase diagram, and show representative configurations of the phase $\mathcal{S}_{d d}$ ). According to the state of art, that is possible only in the strong-coupling regime and at the half-filling.

In the next section we present the model and discuss some of its basic properties. In Section 3, we introduce a strong-coupling expansion of the ground state energy (effective Hamiltonian). In Section 4, we carry out the analysis of phase diagram due to the truncated effective Hamiltonian. Finally, we make conclusions in the Summary.

\section{The model and its basic properties}

The model we consider here, is an extension of the spinless Falicov-Kimball model [10], which in turn is a simplified version of the one introduced in [13. The system is augmented by a direct Ising-like interaction between immobile particles. Hopping particles are allowed to hop not only between n.n. sites (as is considered usually), but between n.n.n. sites as well. Moreover, the n.n.n. hopping intensity depends on direction. The Hamiltonian of the system is of the form:

$$
\begin{aligned}
H_{0} & =H_{F K}+V \\
H_{F K} & =-t \sum_{\langle x, y\rangle_{1}}\left(c_{x}^{+} c_{y}+c_{y}^{+} c_{x}\right)-t_{+} \sum_{\langle x, y\rangle_{2},+}\left(c_{x}^{+} c_{y}+c_{y}^{+} c_{x}\right)-t_{-} \sum_{\langle x, y\rangle_{2},-}\left(c_{x}^{+} c_{y}+c_{y}^{+} c_{x}\right) \\
& +U \sum_{x}\left(c_{x}^{+} c_{x}-\frac{1}{2}\right) s_{x},
\end{aligned}
$$




$$
V=\frac{W}{8} \sum_{\langle x, y\rangle_{1}} s_{x} s_{y}
$$

The underlying lattice $\Lambda$ consists of $|\Lambda|$ sites denoted $x, y, \ldots$, and having the shape of a $\sqrt{|\Lambda|} \times \sqrt{|\Lambda|}$ torus. The sum $\sum_{\langle x, y\rangle_{1}}$ stands for summation over all n.n. pairs, while the sums $\sum_{\langle x, y\rangle_{2,+}}, \sum_{\langle x, y\rangle_{2,-}}-$ for summation over all n.n.n. pairs oriented in the direction with slope $+1(+)$ or in the direction with slope $-1(-)$, respectively.

The subsystem of quantum hopping particles (here-after called the electrons) is described in terms of creation and annihilation operators of an electron at site $x, c_{x}^{+}, c_{x}$, respectively, satisfying the canonical anticommutation relations. The total electron number, $N_{e}$, is the eigenvalue of $\sum_{x} c_{x}^{+} c_{x}$, and the corresponding electron density is $\rho_{e}=N_{e} /|\Lambda|$. Although the electrons do not interact directly with each other, there is an on site interaction with the localized particles that brings energy $U$ if a site is occupied by the both kinds of particles.

Since the site-occupation-number operators of classical immobile particles (here-after called the ions) commute with Hamiltonian (11), the subsystem of ions can be described by a set of pseudo-spins $\left\{s_{x}\right\}_{x \in \Lambda}$ (the ion configurations), with $s_{x}= \pm 1$ ( 1 for the occupied site and -1 for the empty site). The total number of ions is $N_{i}=\sum_{x}\left(s_{x}+1\right) / 2$ and the ion density is $\rho_{i}=N_{i} /|\Lambda|$. In our model the ions interact directly via a n.n. Ising-like interaction $V$, which is isotropic.

The Hamiltonian $H_{F K}$ is the well-known spinless Hamiltonian of the Falicov-Kimball model. A review of rigorous results and an extensive list of references concerning this model can be found in 14, 15, 16].

We shall study the ground-state phase diagram of this system using the grand-canonical formalism, i.e. we consider

$$
H\left(\mu_{e}, \mu_{i}\right)=H_{0}-\mu_{e} N_{e}-\mu_{i} N_{i}
$$

where $\mu_{e}, \mu_{i}$ are the chemical potentials of electrons and ions, respectively. Let $E_{S}\left(\mu_{e}, \mu_{i}\right)$ be the groundstate energy of $H\left(\mu_{e}, \mu_{i}\right)$, for a given configuration $S$ of the ions. Then, the ground-state energy of $H\left(\mu_{e}, \mu_{i}\right), E_{G}\left(\mu_{e}, \mu_{i}\right)$, is defined as $E_{G}\left(\mu_{e}, \mu_{i}\right)=\min \left\{E_{S}\left(\mu_{e}, \mu_{i}\right): S\right\}$. The minimum is attained at the set $G$ of the ground-state configurations of ions.

Applying unitary transformations to $H\left(\mu_{e}, \mu_{i}\right)$ we can restrict the range of the energy parameters. Firstly, using the hole-particle transformation for ions, $s_{x} \rightarrow-s_{x}$, one finds that the case of attraction $(U>0)$ and that of repulsion $(U<0)$ are related by this transformation: if $S=\left\{s_{x}\right\}_{x \in \Lambda}$ is a groundstate configuration at $\left(t, t_{+}, t_{-}, \mu_{e}, \mu_{i}, U\right)$, then $-S=\left\{-s_{x}\right\}_{x \in \Lambda}$ is the ground-state configuration at $\left(t, t_{+}, t_{-}, \mu_{e},-\mu_{i},-U\right)$. Consequently, one can fix the sign of the coupling constant $U$ without any loss of generality. We choose $U>0$. We express also all the other parameters of the Hamiltonian (4) in the units of $U$, i.e. formally we set $U=1$, preserving previous notations.

Secondly, applying the hole-particle transformation for electrons, i.e. $c_{x} \rightarrow \varepsilon_{x} c_{x}^{+}$(where $\varepsilon_{x}$ is equal to +1 on the even sublattice, and -1 on the odd one), and the hole-particle transformation for ions, one finds that if $S$ is the ground-state configuration at $\left(t, t_{+}, t_{-}, \mu_{e}, \mu_{i}\right)$, then $-S$ is the ground-state configuration at $\left(t,-t_{+},-t_{-},-\mu_{e},-\mu_{i}\right)$. Thus, it is enough to consider only one sign of n.n.n. hopping. We shall consider the case of positive n.n.n. hopping intensities: $t_{+}, t_{-}>0$.

Thirdly, using another hole-particle transformation for electrons, $c_{x} \rightarrow c_{x}^{+}$, and the hole-particle transformation for ions, we obtain that if $S$ is the ground-state configuration at $\left(t, t_{+}, t_{-}, \mu_{e}, \mu_{i}\right)$, then $-S$ is the ground-state configuration at $\left(-t,-t_{+},-t_{-},-\mu_{e},-\mu_{i}\right)$. Applying consecutively the two joint (with respect to electrons and ions) hole-particle transformations, we obtain that if $S$ is the groundstate configuration at $\left(t, t_{+}, t_{-}, \mu_{e}, \mu_{i}\right)$, it is also the ground-state configuration at $\left(-t, t_{+}, t_{-}, \mu_{e}, \mu_{i}\right)$. So the relative sign of n.n. and n.n.n. hopping amplitudes does not play any role in the model.

Finally, let us note that Hamiltonian (41), in contrast to that where only n.n. hopping is present, is not invariant with respect to the joint hole-particle transformation for electrons and ions for any values of $\mu_{e}$ and $\mu_{i}$. 


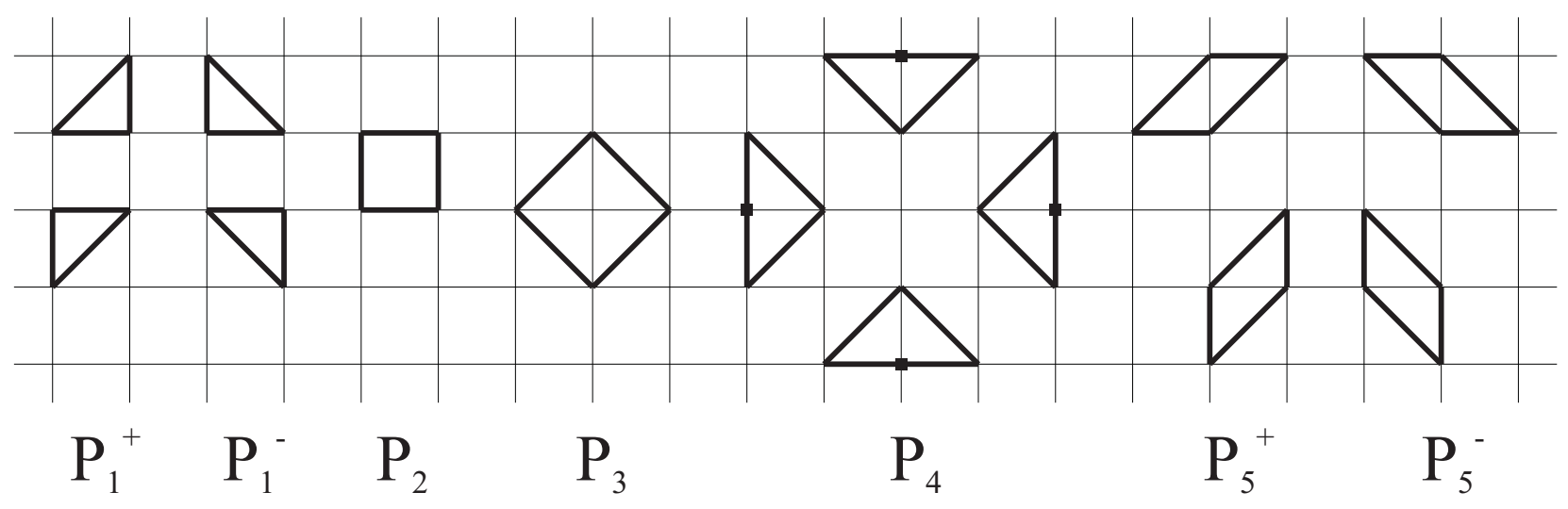

Figure 2: The set of plaquettes (paths) over which the sums are taken in the ground-state energy expansion (5).

\section{The strong-coupling expansion of the ground-state energy}

Using the method of unitary-equivalent interactions [17, in the strong-coupling regime and at half-filling we can expand the ground-state energy $E_{S}$ into a power series in $t^{a} t_{+}^{b} t_{-}^{c}$. The result, with the expansion terms up to the fourth order (the fourth-order effective Hamiltonian), i.e. $a+b+c \leqslant 4$, reads:

$$
\begin{aligned}
E_{S}\left(\mu_{e}, \mu_{i}\right)= & E_{S}^{(4)}\left(\mu_{e}, \mu_{i}\right)+R^{(4)} \\
E_{S}^{(4)}\left(\mu_{e}, \mu_{i}\right)=- & {\left[\frac{1}{2}\left(\mu_{i}-\mu_{e}\right)+\frac{3}{4} t^{2}\left(t_{+}+t_{-}\right)\right] \sum_{x} s_{x}+} \\
& +\left[\frac{1}{4} t^{2}-\frac{9}{16} t^{4}-\frac{1}{16} t^{2}\left(3 t_{+}^{2}+10 t_{+} t_{-}+3 t_{-}^{2}\right)+\frac{W}{8}\right] \sum_{\langle x, y\rangle_{1}} s_{x} s_{y}+ \\
& +\left[\frac{1}{4} t_{+}^{2}+\frac{3}{16} t^{4}-\frac{3}{8} t^{2}\left(2 t_{+}^{2}+t_{+} t_{-}\right)-\frac{3}{16} t_{+}^{4}-\frac{3}{8} t_{+}^{2} t_{-}^{2}\right] \sum_{\langle x, y\rangle_{2,+}} s_{x} s_{y}+ \\
& +\left[\frac{1}{4} t_{-}^{2}+\frac{3}{16} t^{4}-\frac{3}{8} t^{2}\left(2 t_{-}^{2}+t_{+} t_{-}\right)-\frac{3}{16} t_{-}^{4}-\frac{3}{8} t_{+}^{2} t_{-}^{2}\right] \sum_{\langle x, y\rangle_{2},-} s_{x} s_{y}+ \\
& +\left[\frac{1}{8} t^{4}-\frac{1}{8} t^{2} t_{+} t_{-}+\frac{3}{16} t_{+}^{2} t_{-}^{2}\right] \sum_{\langle x, y\rangle_{3}} s_{x} s_{y}+\frac{3}{16} t^{2} t_{+}^{2} \sum_{\langle x, y\rangle_{4},+} s_{x} s_{y}+\frac{3}{16} t^{2} t_{-}^{2} \sum_{\langle x, y\rangle_{4},-} s_{x} s_{y}+ \\
& +\frac{1}{8} t_{+}^{4} \sum_{\langle x, y\rangle_{5,+}} s_{x} s_{y}+\frac{1}{8} t_{-}^{4} \sum_{\langle x, y\rangle_{5,-}} s_{x} s_{y}+\frac{3}{8} t^{2} t_{+} \sum_{P_{1}^{+}} s_{P_{1}^{+}}+\frac{3}{8} t^{2} t_{-} \sum_{P_{1}^{-}} s_{P_{1}^{-}}+ \\
& +\frac{5}{16}\left[t^{4}+2 t^{2} t_{+} t_{-}\right] \sum_{P_{2}} s_{P_{2}}+\frac{5}{16} t_{+}^{2} t_{-}^{2} \sum_{P_{3}} s_{P_{3}}+\frac{5}{16} t^{2} t_{+} t_{-} \sum_{P_{4}} s_{P_{4}}+ \\
& +\frac{5}{16} t^{2} t_{+}^{2} \sum_{P_{5}^{+}} s_{P_{5}^{+}}+\frac{5}{16} t^{2} t_{-}^{2} \sum_{P_{5}^{-}} s_{P_{5}^{-}} .
\end{aligned}
$$

Here, the sign $\bullet$ in the $\langle x, y\rangle_{i, \bullet}$ means a positive or a negative slope of an $i$-th order n.n. pair. The sets of plaquettes (or paths) $P_{i}^{\bullet}$ are shown in Fig. 2. The sign $\bullet$ in the superscript of the path $P_{i}^{\bullet}$ reflects the sign of the slope of the n.n.n. pairs in it. The remainder $R^{(4)}$ is independent of the chemical potentials and $W$, and collects all the terms proportional to $t^{a} t_{+}^{b} t_{-}^{c}$, with $a+b+c=5,6, \ldots$. The above expansion is absolutely convergent for sufficiently small $t, t_{+}$and $t_{-}$, uniformly in $\Lambda$. In the special case of $t_{+}=t_{-}=t^{\prime}$ and $W=0$ it was obtained in [18].

Let us note that the ground-state energy expansion (515) depends only on $\left(\mu_{i}-\mu_{e}\right)$. Hence, we denote 
this difference as the unique chemical potential parameter $\mu$.

In Ref. [8], we have obtained the phase diagram of the isotropic model without n.n.n. hopping, i.e. for $t_{+}=t_{-}=0$. Here, our aim is to determine the influence of the n.n.n.-hopping anisotropy on the diagonal-striped phase $\mathcal{S}_{d d}$ (see Fig. 1). For this job, the value of the n.n.n.-hopping intensities, $t_{+}, t_{-}$, cannot be too large, in order to preserve the phase diagram up to 4 th order. On the other hand, the n.n.n.-hopping intensities cannot be too small, in order to appear in the fourth-order effective Hamiltonian. In an attempt to satisfy the both requirements, we choose the smallest n.n.n.-hopping intensities $t_{+}, t_{-}$, i.e. such that they do not appear in the expansion terms of order smaller than four: $t_{+}=a_{+} t^{2}$ and $t_{-}=a_{-} t^{2}$. In this case, the effective Hamiltonian assumes the form:

$$
\begin{aligned}
E_{S}^{(4)}(\mu)= & -\left[\frac{1}{2} \mu+\frac{3}{4} t^{4}\left(a_{+}+a_{-}\right)\right] \sum_{x} s_{x}+\left[\frac{1}{4} t^{2}-\frac{9}{16} t^{4}+\frac{W}{8}\right] \sum_{\langle x, y\rangle_{1}} s_{x} s_{y}+ \\
& +\left[\frac{1}{4} t^{4} a_{+}^{2}+\frac{3}{16} t^{4}\right] \sum_{\langle x, y\rangle_{2},+} s_{x} s_{y}+\left[\frac{1}{4} t^{4} a_{-}^{2}+\frac{3}{16} t^{4}\right] \sum_{\langle x, y\rangle_{2},-} s_{x} s_{y}+ \\
& +\frac{1}{8} t^{4} \sum_{\langle x, y\rangle_{3}} s_{x} s_{y}+\frac{3}{8} t^{4} a_{+} \sum_{P_{1}^{+}} s_{P_{1}^{+}}+\frac{3}{8} t^{4} a_{-} \sum_{P_{1}^{-}} s_{P_{1}^{-}}+\frac{5}{16} t^{4} \sum_{P_{2}} s_{P_{2}},
\end{aligned}
$$

i.e. the second requirement is satisfied. To answer the question put in the introduction, concerning the influence of anisotropy of n.n.n. hopping on the degeneracy of the phase $\mathcal{S}_{d d}$, there is no need to consider the whole phase diagram. For $t_{+}=t_{-}=0$, we fix a point, well inside the domain of the diagonal-striped phase $S_{d d}$, say $\mu=0$ and $W=-2 t^{2}+9 / 2 t^{4}$, i.e. $\omega=9 / 2$ (see Fig. 1). Then, with the fixed point in $(\mu, W)$-plain, we introduce a n.n.n. hopping which does not change the ground-state configurations. Calculations show that $\mathcal{S}_{d d}$ has the minimal energy for $a=\left|a_{+}\right|=\left|a_{-}\right|$, where $-1 / 4 \leqslant a \leqslant 1 / 4$ (we suppose that the difference between $a_{+}$and $a_{-}$is not large, so they are of the same sign). Therefore, with our choice of n.n.n.-hopping intensities, the first of the above two requirements can also be satisfied. Eventually, we fix the values of n.n.n.-hopping intensities: $a_{+}=1 / 8, a_{-}=\gamma a_{+}$, with $\gamma$ varying about 1 (say, $0 \leqslant \gamma \leqslant 2$ ).

Since all the energy parameters, except the parameter $\gamma$ of n.n.n.-hopping anisotropy, have been fixed, the effective Hamiltonian (6) depends only on $\gamma$. In the following section, we examine how n.n.n.-hopping anisotropy, $\gamma \neq 1$, influences the degeneracy of the diagonal-striped phase $\mathcal{S}_{d d}$.

\section{Diagonal-striped phase versus n.n.n.-hopping anisotropy}

We use the $m$-potential method [19] for constructing the phase diagram of effective Hamiltonian $E_{S}^{(4)}(\gamma)$. For technical reasons, it is convenient to deal with such energies of configurations that are affine functions of the parameters of the effective Hamiltonian. However, the effective Hamiltonian (6) contains the terms proportional to $\gamma$ and $\gamma^{2}$. To get rid of nonlinearities, we replace $\gamma$ and $\gamma^{2}$ by two independent parameters $d_{1}$ and $d_{2}$, respectively, with $d_{1}, d_{2}$ varying in the rectangle $0 \leq d_{1} \leq 2$ and $0 \leq d_{2} \leq 4$, in which the Hamiltonian is affine. After constructing the phase diagram in $\left(d_{1}, d_{2}\right)$-plane, we restrict it to the $d_{2}=d_{1}^{2}$ curve.

To compare the energies of configurations, we rewrite $E_{S}^{(4)}\left(d_{1}, d_{2}\right)$ as the sum,

$$
E_{S}^{(4)}\left(d_{1}, d_{2}\right)=\frac{t^{4}}{2} \sum_{T} H_{T}^{(4)},
$$


over $(3 \times 3)$-site blocks (called $T$-plaquettes). The potential $H_{T}^{(4)}$ is of the form:

$$
\begin{aligned}
H_{T}^{(4)}= & -\frac{3}{16}\left(d_{1}+1\right) s_{5}+\frac{49}{512} \sum_{\langle x, y\rangle_{2},+}^{\prime \prime} s_{x} s_{y}+\frac{1}{32}\left(\frac{1}{16} d_{2}+3\right) \sum_{\langle x, y\rangle_{2},-}^{\prime \prime} s_{x} s_{y}+\frac{1}{12} \sum_{\langle x, y\rangle_{3}}^{\prime \prime} s_{x} s_{y}+ \\
& +\frac{3}{128} \sum_{P_{1}^{+}}^{\prime \prime} s_{P_{1}^{+}}+\frac{3}{128} d_{1} \sum_{P_{1}^{-}}^{\prime \prime} s_{P_{1}^{-}}+\frac{5}{32} \sum_{P_{2}}^{\prime \prime} s_{P_{2}},
\end{aligned}
$$

where $s_{5}$ is the central site of a $T$-plaquette.

Unfortunately, the potential (8) is not an $m$-potential in the rectangle of considered values of $d_{1}$ and $d_{2}$. Therefore, following [20, 21, 22] we introduce so-called zero-potentials. The zero potentials, satisfying

$$
\sum_{T} K_{T}^{(4)}=0
$$

can be chosen in the form:

$$
K_{T}=\sum_{i=1}^{9} \alpha_{i} k_{T}^{(i)}
$$

where coefficients $\alpha_{i}$ have to be determined in the process of constructing a phase diagram, and the potentials $k_{T}^{(i)}$, invariant with respect to the spatial symmetries of $H_{0}$, and fulfilling (91), read:

$$
\begin{aligned}
& k_{\mathrm{T}}^{(1)}=s_{1}+s_{9}-2 s_{5}, \\
& k_{\mathrm{T}}^{(2)}=s_{2}+s_{8}-2 s_{5}, \\
& k_{\mathrm{T}}^{(3)}=s_{3}+s_{7}-2 s_{5}, \\
& k_{\mathrm{T}}^{(4)}=s_{4}+s_{6}-2 s_{5}, \\
& k_{\mathrm{T}}^{(5)}=s_{1} s_{2}+s_{8} s_{9}-s_{4} s_{5}-s_{5} s_{6}, \\
& k_{\mathrm{T}}^{(6)}=s_{2} s_{3}+s_{7} s_{8}-s_{4} s_{5}-s_{5} s_{6}, \\
& k_{\mathrm{T}}^{(7)}=s_{1} s_{4}+s_{6} s_{9}-s_{2} s_{5}-s_{5} s_{8}, \\
& k_{\mathrm{T}}^{(8)}=s_{3} s_{6}+s_{4} s_{7}-s_{2} s_{5}-s_{5} s_{8}, \\
& k_{\mathrm{T}}^{(9)}=s_{2} s_{4}+s_{6} s_{8}-s_{3} s_{5}-s_{5} s_{7} .
\end{aligned}
$$

Here, we label $1, \ldots, 9$, the sites of a $T$-plaquette, from left to right, starting in the bottom left corner and ending in the upper right one. In order to obtain the phase diagram, we have to compare the energies of all the possible $T$-plaquette configurations. The zero-potential coefficients $\alpha$ needed for this are given in Tab. 1 in the Appendix. We provide their values only at certain generating points, since we can assume that the coefficients $\alpha$ are affine functions of parameters $\left(d_{1}, d_{2}\right)$. For more details on using the $m$-potential method see 12 and references therein.

The phase diagram of $E^{(4)}$ is shown in Fig. 3. The rectangle of considered points $\left(d_{1}, d_{2}\right)$ breaks down into two domains. In the lower one, where $\gamma<1$ and $t_{-}<t_{+}$, it is the phase $\mathcal{S}_{d d}^{-}$, with stripes being parallel to the direction of $t_{-}$-hopping, that is stable. The analogous situation is in the upper domain, where $\gamma>1$ and $t_{-}>t_{+}$: the stable phase, $\mathcal{S}_{d d}^{+}$, consists of stripes oriented along $t_{+}$-hopping. At $\gamma=1$, we have the isotropic phase $\mathcal{S}_{d d}$ whose configurations consist of $\mathcal{S}_{d d}^{+}$and $\mathcal{S}_{d d}^{-}$.

So we see that, at least for the truncated effective Hamiltonian, switching on of a n.n.n.-hopping anisotropy reduces the rotational degeneracy of diagonal-striped phases: they become oriented in the direction of the weaker hopping. 


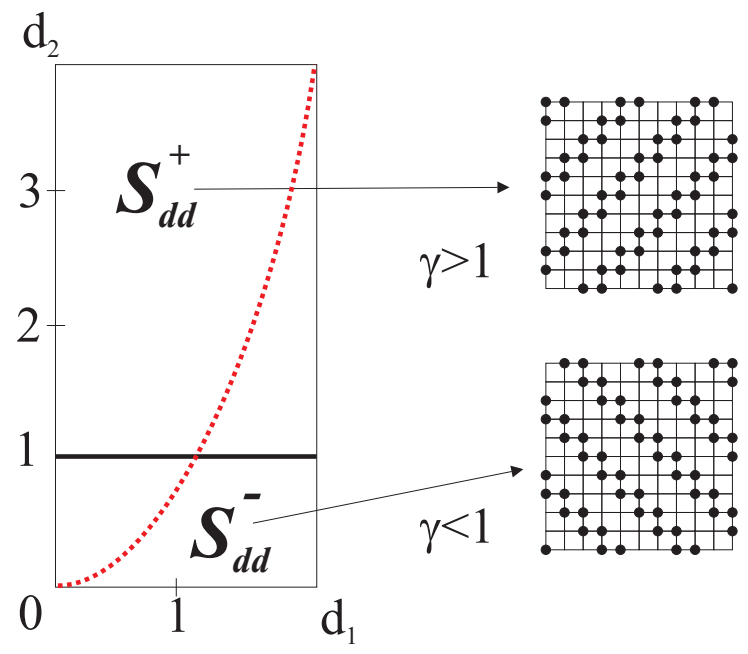

Figure 3: The phase diagram of $E^{(4)}\left(d_{1}, d_{2}\right)$, with $0 \leqslant d_{1} \leqslant 2$ and $0 \leqslant d_{2} \leqslant 4$. The rectangle breaks down into two regions: above $d_{2}=1$ the configurations $\mathcal{S}_{d d}^{+}$are the ground-state configurations, while $\mathcal{S}_{d d}^{+}$are replaced by $\mathcal{S}_{d d}^{-}$below $d_{2}=1$. The dashed line represents the condition $d_{2}=d_{1}^{2}$. The representative configurations (up to translations) of $\mathcal{S}_{d d}^{+}, \mathcal{S}_{d d}^{-}$are shown on the right.

This result is similar to that described in [12, where the influence of n.n.-hopping anisotropy on axial-striped phases was investigated. In that case, not only for a truncated effective Hamiltonian but also for the corresponding quantum one, it was proved that for any nonzero value of n.n.-hopping anisotropy the rotational degeneracy of axial-striped phases is reduced by making them oriented along the direction of the weaker n.n. hopping. Now in turn, the natural question is whether the conclusions we arrived at, concerning n.n.n.-hopping anisotropy, hold true for the quantum model, described by Hamiltonian (4). Applying the arguments presented in [21, 22], we can demonstrate (see for instance Ref. [23]), that the stable phases of the obtained above phase diagram remain stable for the model (4), but in some smaller domains. That is, if the remainder $R^{(4)}$ is taken into account, then there exist such a small $t_{0}$, that for $t<t_{0}$ the phase diagram looks the same for the quantum model, excepting of some narrow regions (of width $O(t)$ in the scale of the fourth-order phase diagram shown in Fig. 33), located along the phase-boundary lines. In our case that means that the breaking of the rotational symmetry occurs for $\gamma=1+O(t)$, when the n.n.n.-hoping intensities are $O\left(t^{2}\right)$. Unfortunately, we cannot claim that any non-zero n.n.n.-hopping anisotropy reduces the rotational degeneracy of the quantum model, as it was the case for n.n. hopping (see [12]). Here it seems, at least for small n.n.n.-hopping intensities, that there is certain critical value of $|\gamma-1|$, above which the degeneracy of phase $\mathcal{S}_{d d}$ is reduced.

\section{Summary}

We have considered the model of correlated spinless fermions, described by an extended FalicovKimball Hamiltonian. Quantum particles are allowed to hop between nearest-neighbor and next-nearestneighbor sites. We have shown that a weak anisotropy of the next-nearest-neighbor hopping reduces the degeneracy of a diagonal-striped phase, it orients the stripes in the direction of the weaker nextnearest-neighbor hopping.

\section{Acknowledgments}

The author appreciates Prof. Janusz Jędrzejewski's valuable and fruitful discussions as well as critical review of the manuscript and thoughtful suggestions. 
The author is grateful to University of Wrocław for Scientific Research Grant 2479/W/IFT, and to the Institute of Theoretical Physics for financial support. The Max Born Scholarship is gratefully acknowledged.

\section{References}

[1] J.M. Tranquada, D.J. Buttrey, V. Sachan, and J.E. Lorenzo, Simultaneous Ordering of Holes and Spins in $\mathrm{La}_{2} \mathrm{NiO}_{4.125}$, Phys. Rev. Lett. 73, 1003 (1994)

[2] J.M. Tranquada, B.J. Sternlieb, J.D. Axe, Y. Nakamura, and S.Uchida, Evidence for stripe correlations of spins and holes in copper oxide superconductors, Nature (London) 375, 561 (1995)

[3] A.M. Oleś, Stripe phases in high-temperature superconductors, Acta Phys. Polonica B 31, 2963 $(2000)$

[4] N.G. Zhang and C.L. Henley, Stripes and holes in a two-dimensional model of spinless fermions or hardcore bosons, Phys. Rev. B 68, 014506 (2003)

[5] C.L. Henley and N.G. Zhang, Spinless fermions and charged stripes at the strong-coupling limit, Phys. Rev. B 63, 233107 (2001)

[6] R. Lemański, J.K. Freericks, and G. Banach, Stripe Phases in the Two-Dimensional Falicov-Kimball Model, Phys. Rev. Lett. 89, 196403 (2002)

[7] R. Lemański, J.K. Freericks, and G. Banach, Charge Stripes due to Electron Correlations in the Two-Dimensional Spinless Falicov-Kimball Model, J. Stat. Phys. 116, 699 (2004)

[8] V. Derzhko and J. Jędrzejewski, Formation of charge-stripe phases in a system of spinless fermions or hardcore bosons, Physica A 349, 511 (2005).

[9] U. Brandt, R. Schmidt, Exact results for the distribution of the f-level ground state occupation in the spinless Falicov-Kimball model, Z. Phys. B 63, 45 (1986).

[10] T. Kennedy and E. H. Lieb, An itinerant electron model with crystalline or magnetic long range order, Physica A 138, 320 (1986).

[11] M. Raczkowski, B. Normand, and A. M. Oleś, Vertical and diagonal stripes in the extended Hubbard model, Phys. Stat. Sol. (b) 236, 376 (2003)

[12] V. Derzhko and J. Jȩdrzejewski, Charge-stripe phases versus a weak anisotropy of nearest-neighbor hopping, ArXiv:cond-mat/0509698.

[13] L.M. Falicov and J.C. Kimball, Simple model for semiconductor-metal transitions: $S_{m} B_{6}$ and transition-metal oxides, Phys. Rev. Lett. 22, 997 (1969).

[14] J. Jȩdrzejewski and R. Lemański, Falicov-Kimball models of collective phenomena in solids (a concise guide), Acta Phys. Pol. B 32, 3243 (2001).

[15] C. Gruber and N. Macris, The Falicov-Kimball model: a review of exact results and extensions, Helv. Phys. Acta 69, 850 (1996).

[16] C. Gruber and D. Ueltschi, The Falicov-Kimball model, arXiv:math-ph/0502041.

[17] N. Datta, R. Fernández, and Jürg Fröhlich, Effective Hamiltonians and phase diagrams for tightbinding models, J. Stat. Phys. 96, 545 (1999). 
[18] J. Wojtkiewicz, Phase diagram of the two-dimensional $t$ - $t^{\prime}$ Falicov-Kimball model, arXiv:cond-mat/0310043.

[19] J. Slawny, Low-temperature properties of classical lattice systems: phase transitions and phase diagrams, In: Phase Transitions and Critical Phenomena vol. 11, C. Domb and J. Lebowitz, eds (Academic Press, London/New York 1985).

[20] C. Gruber, J. Jȩdrzejewski and P. Lemberger, Ground states of the spinless Falicov-Kimball model. II, J. Stat. Phys. 66, 913 (1992).

[21] C. Gruber, N. Macris, A. Messager and D. Ueltschi, Ground states and flux configurations of the two-dimensional Falicov-Kimball model, J. Stat. Phys. 86, 57 (1997).

[22] T. Kennedy, Some rigorous results on the ground states of the Falicov-Kimball model, Rev. Math. Phys. 6, 901 (1994).

[23] V. Derzhko and J. Jȩdrzejewski, From phase separation to long-range order in a system of interacting electrons, Physica A 328, 449 (2003)

\section{Appendix}

Here we present the zero-potential coefficients $\alpha_{i}$ in the generating points of phase diagram shown in Fig. 3.

Table 1: Zero-potentials coefficients for the phase diagram shown in Fig. 3

\begin{tabular}{|l|c|c|c|c|c|c|}
\hline Points $\left(d_{1}, d_{2}\right)$ & $(0,0)$ & $(2,0)$ & $(0,1)$ & $(2,1)$ & $(0,4)$ & $(2,4)$ \\
\hline$\alpha_{1}$ & $-\frac{83}{1536}$ & $-\frac{259}{3072}$ & $-\frac{79}{1536}$ & $-\frac{253}{3072}$ & $-\frac{67}{1536}$ & $-\frac{217}{3072}$ \\
\hline$\alpha_{2}$ & $-\frac{25}{1536}$ & $-\frac{245}{3072}$ & $-\frac{29}{1536}$ & $-\frac{251}{3072}$ & $-\frac{41}{1536}$ & $-\frac{287}{3072}$ \\
\hline$\alpha_{3}$ & $-\frac{47}{1536}$ & $-\frac{331}{3072}$ & $-\frac{43}{1536}$ & $-\frac{325}{3072}$ & $-\frac{31}{1536}$ & $-\frac{289}{3072}$ \\
\hline$\alpha_{4}$ & $-\frac{25}{1536}$ & $-\frac{245}{3072}$ & $-\frac{29}{1536}$ & $-\frac{251}{3072}$ & $-\frac{41}{1536}$ & $-\frac{287}{3072}$ \\
\hline$\alpha_{5}$ & $\frac{53}{3072}$ & $\frac{137}{8192}$ & $\frac{19}{1024}$ & $\frac{139}{8192}$ & $\frac{3}{128}$ & $\frac{91}{8192}$ \\
\hline$\alpha_{6}$ & $-\frac{53}{3072}$ & $-\frac{335}{24576}$ & $-\frac{19}{1024}$ & $-\frac{365}{24576}$ & $-\frac{3}{128}$ & $-\frac{91}{8192}$ \\
\hline$\alpha_{7}$ & $\frac{53}{3072}$ & $\frac{401}{8192}$ & $\frac{19}{1024}$ & $\frac{403}{8192}$ & $\frac{3}{128}$ & $\frac{1117}{24576}$ \\
\hline$\alpha_{8}$ & $-\frac{53}{3072}$ & $-\frac{571}{24576}$ & $-\frac{19}{1024}$ & $-\frac{589}{24576}$ & $-\frac{3}{128}$ & $-\frac{139}{6144}$ \\
\hline$\alpha_{9}$ & $-\frac{19}{3072}$ & $\frac{101}{2048}$ & $-\frac{19}{3072}$ & $\frac{13}{256}$ & $-\frac{19}{3072}$ & $\frac{1451}{24576}$ \\
\hline
\end{tabular}

